\title{
Audience Interest Analysis based on the Feedback of IPTV Users
}

\author{
Anu Joseph \\ M.Tech, Computer Science and Information \\ Systems, FISAT Engineering College, Kerala
}

\author{
Hema Krishnan \\ PhD Scholar, Assistant Professor, FISAT \\ Engineering College, Kerala
}

\begin{abstract}
The assessment and analysis of public opinion and people's interest in various topics have been highly important for decades. Every major social, economic or political decision process relies on tapping the pulse of the public opinion through time, and tries to adjust based on the feedback. In this work the focus is on channel change events (CCE) generated by the viewers. CCE data can be represented by a time series vector; it hides a wealth of user behavior information, as each channel change event is motivated by a combination of viewers interests and content context. The key challenge addressed in the paper is to demonstrate how the users inter actions with the IPTV service can be efficiently used to gauge the public interest on a specific topic at a large scale. To address the challenges of using an implicit feedback event stream of an IPTV system to infer public interest and opinion on a large scale, proposes a framework that leverages a variety of research domain.
\end{abstract}

\section{Keywords}

Individuals, mining, input, curation, assessing, IPTV, channel change, responses

\section{INTRODUCTION}

Internet Protocol Television (IPTV) is a framework through which Internet TV administrations are conveyed utilizing the design and systems administration techniques for the Internet Convention Suite over a bundle exchanged system foundation e.g the Internet and broadband Internet get to systems, rather than being conveyed through customary radio recurrence communicate, satellite sign, and digital TV designs. TV experiences an extreme change from unidirectional telecom to Internet-associated. The new test is specifically featured in how to convey a universe of substance to any gadget, whenever, anyplace .Internet Protocol Television (IPTV) is equipped for conveying rich set administrations alongside excellent TV content through Internet Protocol (IP) systems. The utilization of IP organizing suggests that media situated in diff erent sources can be conveyed over any IP system to reach an expansive number of customers. In addition, the shopper can re-collect the media substance to fulfil his very own necessities utilizing privately executed programming and personalization engines. The IPTV administration is conveyed to buying in purchasers over a broadband association with a set-top box.

\subsection{IPTV Viewership}

Activities of IPTV viewership off er an engaging option in contrast to online client generated content investigation with some significant focal points. To begin with, the number of inhabitants in IPTV gathering of people by and large speaks to a considerably more assorted gathering of individuals, which is all the more consistently dispersed crosswise over diff erent age gatherings. Second, the interests and conclusions are certainly and some of the time even subliminally communicated, without companion weight initiated curation or self-controlling, and without outside blue penciling that would twist the conclusion. Similarly significant is the way that certain gathering of intrigue and conclusion does not upset the nature of experience. Client created occasions (e.g., channel change)occasions) speak to an enormously parallel casting a ballot framework, where clients either sanely or intuitively vote on a scope of themes that are being displayed. Consequently, observing a certain casting a ballot procedure has leeway when contrasted with the unequivocal strategies, which require the client to play out an activity (e.g., answer an inquiry). In the meantime the verifiable procedure lessens oneself editing of the response imprints The accumulation of such information has for quite some time been hazardous and has required specialized frameworks (e.g., redid set-top boxes) conveyed with a set number of clients. Such frameworks have high arrangement and support costs. Nonetheless, as of late pervasive organizations of Internet Protocol TV (IPTV) systems, which are regularly a piece of the Triple Play off erings around the globe, make the accumulation of such information progressively simple.

\subsection{Channel change events}

In this venture the emphasis is on channel change occasions (CCE) produced by the viewers. CCE information can be spoken to by a period arrangement vector; it shrouds an abundance of client conduct data, as each channel change occasion is persuaded by a combination of watcher's interests and substance setting. The key test tended to in the venture is to show how the clients' communications with the IPTV administration can be efficiently used to measure the open enthusiasm on a specific subject at an extensive scale. The principle commitments of this paper are: The proposition of a structure for surveying the clients understood positive and negative input as for the channel change events. Presentation and investigation of a model usage of the depicted system. The actualized technique depends on learning and permits the estimation of the open enthusiasm on a specific theme and examination of intrigue between themes. The proposed methodology can likewise be reached out in various ways to permit progressively expand use cases.

\section{METHODS}

\subsection{The Feature set selection}

The feature set selection part of the framework is the base part of the system. Feature set must choose in such a way that ,it must be fast and efficient to reach a conclusion after performing data analysis on the features. The features collected are uid , main uid , starttime, stoptime , channel, channelid, program etc. 


\subsection{Data Analysis}

Data analysis includes from the chosen features selecting the appropriate information. For example programs with view count less than fifty will be discarded and channels with view count less than hundred will be discarded. For analyzing the user behavior through graphs only consider the chosen information.

\subsection{User Behavior Analysis}

Once the features are collected ,System performs data analysis and based on these data, Predicts the user Interest. Results of these analysis are draw as graphs that is having very much relevance in predicting user behavior. Firstly Compared the analyzed channel change events and the watched channels and Drawn the graph of channel VS channel usage. The total channel view count grater than 100 then only considered that channel for plotting the graph. Secondly Compared the analyzed channel change events and the watched channels and Drawn the graph of Trending Programs VS channel usage. The total Program view count grater than 50 then only considered that channel for plotting the graph. Lastly Compared the analyzed watched channels on monthly basis and plotted the graph of monthly channel usage. The months from oct- mar is considered for plotting the graph of monthly report.

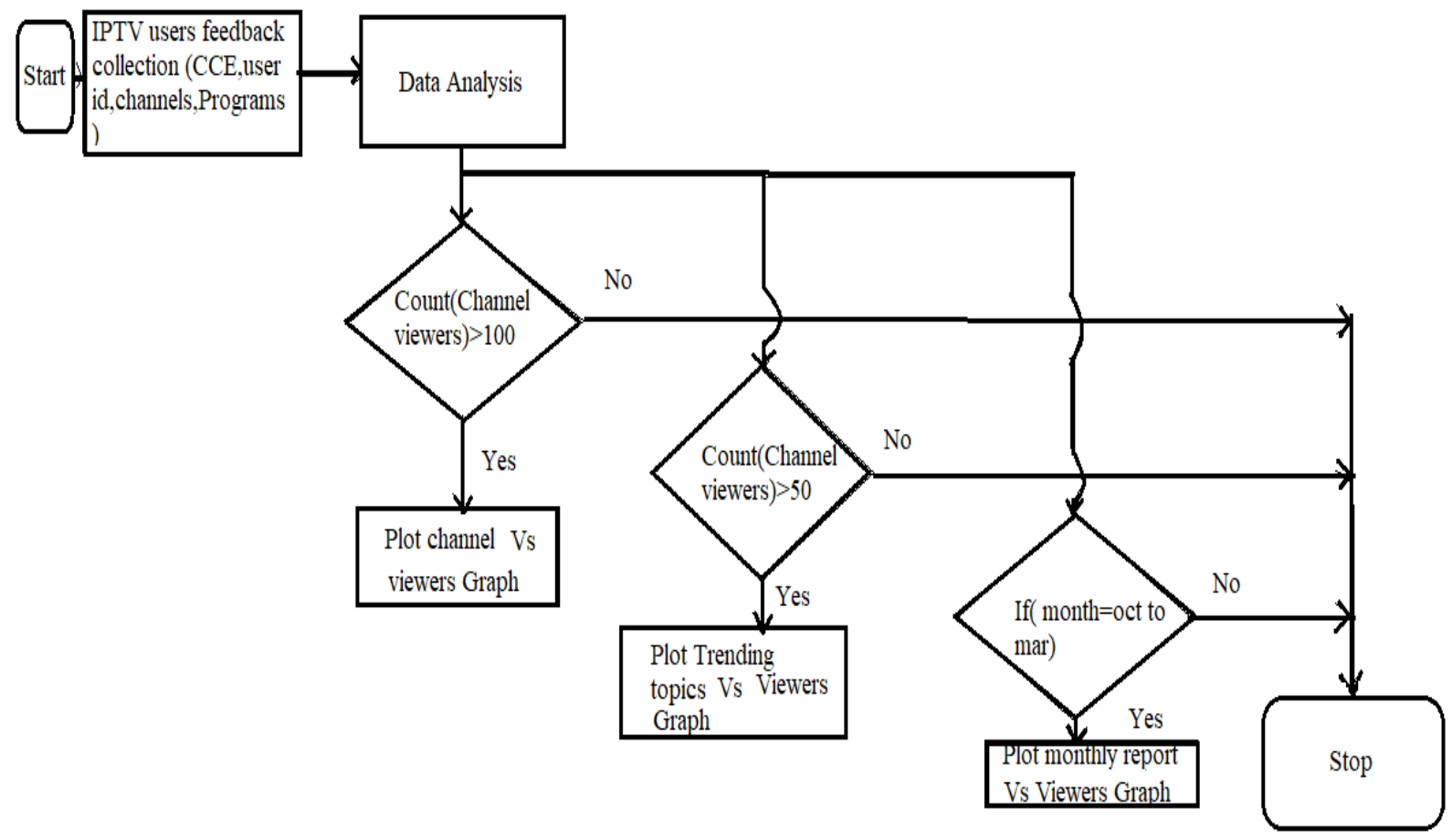

Fig 1: Flow chart of the proposed system

\section{RESULTS AND DISCUSSIONS}

To demonstrate the usability of implementation of the proposed framework collected features from IPTV Dataset. The features collected include uid, main uid, start time, stop time, channel, channelid, program etc. of IPTV Dataset of diff erent time periods are compared and analyzed. Based on these analysis plotted bar graphs. Firstly plotted a graph for analyzed trending channels and it's usage. Then plotted a graph for trending programs and it's viewers. Lastly plotted graph for the time period from October 2011 to march 2012, Analyzed the monthly popularity of watching the channels and found that in the month of November is having high IPTV usage.

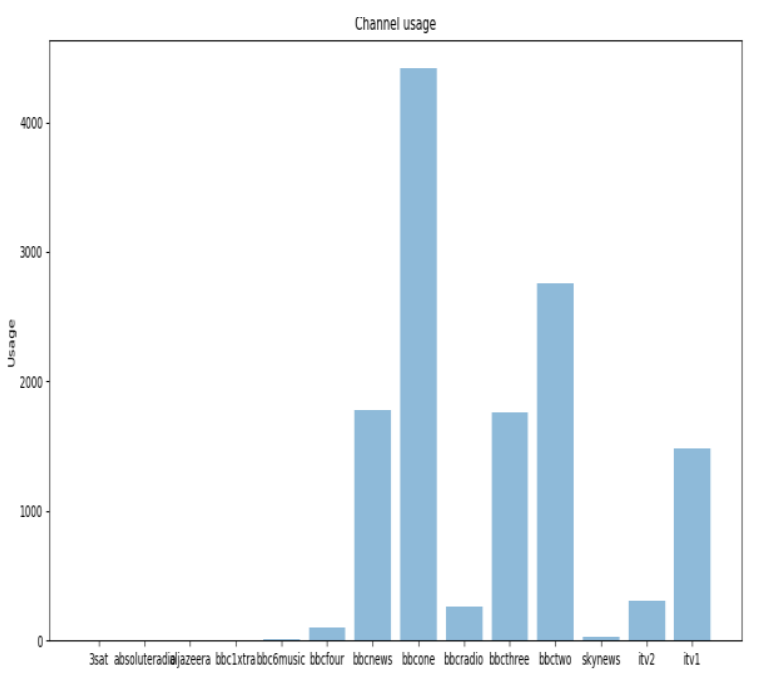

Fig 2: Channel Vs Usage graph 


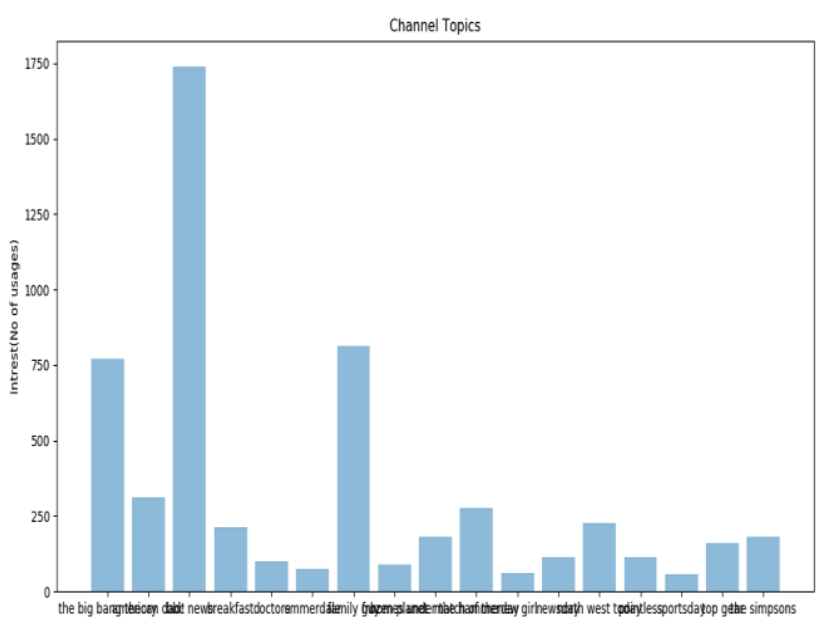

Fig 3: Program Vs usage graph

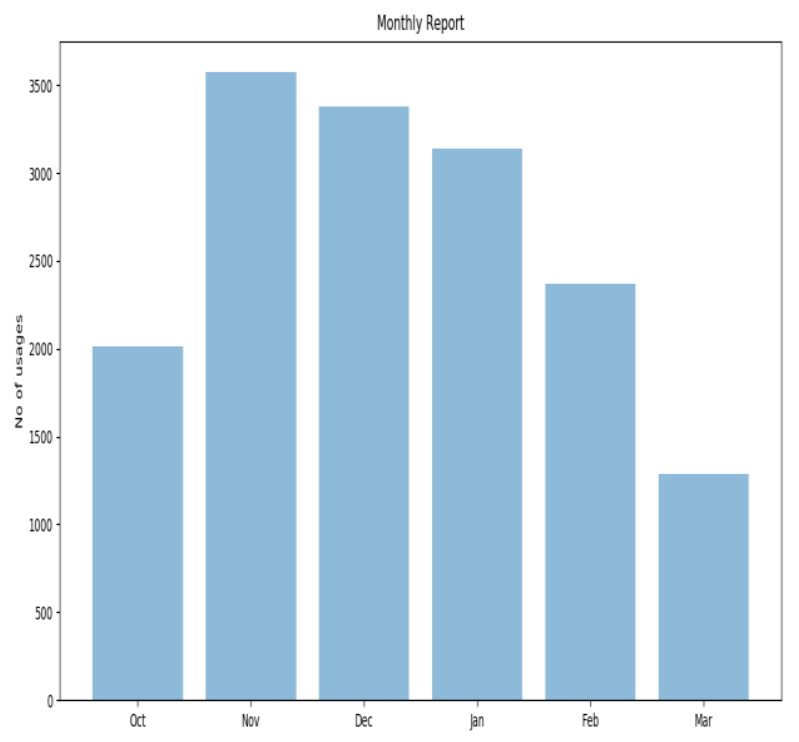

Fig 4: Month Vs usage graph

\section{CONCLUSIONS}

In this project, presented a novel framework for public interest and opinion analysis based on IPTV user behavior. Project presented the basic framework components and provided their desirable properties and functionalities and further described a possible implementation of the framework in detail. The presented Dataset was analyzed and the results showed its usability as the public interest measure. The future work will focus on the crowd-sourced data collection and mining of the IPTV network-based event data. The research will be divided into small segments, described in the framework section. The first segment of the improvement of the system is in the data collection part, where program information will be added for broader range of transmissions. The second part of the future work will focus on improving the language model. The last part of the improvement is analysis of various evaluation algorithms. Another very important task left for future study is the mapping of the implicitly collected public interest measure into a credible and reliable public opinion measure. All of the above mentioned future tasks involve interdisciplinary and multidisciplinary research.

\section{REFERENCES}

[1] Privacy Usability of IPTV Recommender Systems, Tolga Arul, Nikolaos Athanasios Anagnostopoulos, Stefan Katzenbeisser,Security Engineering Group, Department of Computer Science, Technische Universitat Darmstadt

[2] Mining the IPTV Channel Change Event Stream to Discover Insight and Detect Ads, MatejKren, AndrejKos, andUrbanSedlar,Hindawi 2016.

[3] Reliable Gender Prediction Based on Users Video Viewing Behavior ,Jie Zhang, Kuang Du, Ruihua Cheng, Zhi Wei,, Chenguang Qin, Huaxin You, Sha Hu ,2016 IEEE 16th International Conference on Data Mining.

[4] Audiences Viewing Behavior Analysis for Inferencing Consumer Preferences,Sang-Yun Lee, Jeong-Woo Son ,Sun-Joong Kim, Won Ryu,2015 IEEE.

[5] Crowd Mining System for TV Program Based on Audience Behavior Analysis ,Fulian Yin, Lu Lu, You Li, Jianping Chai ,7th International Conference on Advanced Computational Intelligence Mount March 2729,2015 .

[6] Feature Extraction Algorithm and Optimizations for Mass TV Audience,Fan Zhang,Zheng Chen, Jinyao Yan ,International Conference on Cyber-Enabled Distributed Computing and Knowledge Discovery ,2015.

[7] Rating Prediction Algorithm and Recommendation Based on User Beahavior In IPTV,YueTeng,LiangHe,IEEE 2012.

[8] The use of implicit evidence for relevance feedback in web retrieval and Feature Extraction in Sentiment Analysis ,Wani , Ian Ruthven, Joemon M. Jose ,IEEE 2015 international conference.

[9] Mathematical models of peer to peer networks for stream IPTV transmission,Naors Y. Anad Alsaleem ; Riyad Mubarak Abdullah ; Maan Y. AnadAlsaleem, 2018 IEEE 9th International Conference on Dependable Systems, Services and Technologies (DESSERT).

[10] Performance of QOS parameters for IPTV through NGN,Farouk A. Elgeldawy, Gerges M. Salama ; Marwa F. Abdel fattah, 2016 IEEE Student Conference on Research and Development (SCOReD). 